\title{
PraCtICE
}

\section{Diagnosis and treatment of dementia: 2. Diagnosis}

\author{
Howard H. Feldman MD, Claudia Jacova PhD, Alain Robillard MD, Angeles Garcia MD PhD, \\ Tiffany Chow MD, Michael Borrie MB ChB, Hyman M. Schipper MD PhD, Mervin Blair BSc, \\ Andrew Kertesz MD, Howard Chertkow MD
}

\section{ABSTRACT}

Background: Dementia can now be accurately diagnosed through clinical evaluation, cognitive screening, basic laboratory evaluation and structural imaging. A large number of ancillary techniques are also available to aid in diagnosis, but their role in the armamentarium of family physicians remains controversial. In this article, we provide physicians with practical guidance on the diagnosis of dementia based on recommendations from the Third Canadian Consensus Conference on the Diagnosis and Treatment of Dementia, held in March 2006.

Methods: We developed evidence-based guidelines using systematic literature searches, with specific criteria for study selection and quality assessment, and a clear and transparent decision-making process. We selected studies published from January 1996 to December 2005 that pertained to key diagnostic issues in dementia. We graded the strength of evidence using the criteria of the Canadian Task Force on Preventive Health Care.

Results: Of the 1591 articles we identified on all aspects of dementia diagnosis, 1095 met our inclusion criteria; 620 were deemed to be of good or fair quality. From a synthesis of the evidence in these studies, we made 32 recommendations related to the diagnosis of dementia. There are clinical criteria for diagnosing most forms of dementia. A standard diagnostic evaluation can be performd by family physicians over multiple visits. It involves a clinical history (from patient and caregiver), a physical examination and brief cognitive testing. A list of core laboratory tests is recommended. Structural imaging with computed tomography or magnetic resonance imaging is recommended in selected cases to rule out treatable causes of dementia or to rule in cerebrovascular disease. There is insufficient evidence to recommend routine functional imaging, measurement of biomarkers or neuropsychologic testing.

Interpretation: The diagnosis of dementia remains clinically integrative based on history, physical examination and brief cognitive testing. A number of core laboratory tests are also recommended. Structural neuroimaging is advised in se- lected cases. Other diagnostic approaches, including functional neuroimaging, neuropsychological testing and measurement of biomarkers, have shown promise but are not yet recommended for routine use by family physicians.

Une version française de ce résumé est disponible à l'adresse www.cmaj.ca/cgi/content/full/178/7/825/DCI

CMAJ 2008;178(7):825-36

\section{The case}

Mrs. E, a 72-year-old woman in your practice who has experienced clear and progressive memory decline over the past 3 years, finally agrees to come and see you with her daughter and husband for an assessment. The patient is English speaking and has a bachelor's degree. Her medical history includes hypertension, a 20-pack-year history of smoking and a previous transient ischemic attack. Her family says that she is unable to currently handle her own banking and has become lost when driving. Mrs. E is increasingly anxious when left alone. Her husband is concerned that she might be depressed, but he adds

From the Division of Neurology, Department of Medicine, University of British Columbia, and the University of British Columbia Hospital Clinic for Alzheimer's Disease and Related Disorders (Feldman, Jacova), Vancouver, BC; the Division of Neurology, Department of Medicine (Robillard), Hôpita Maisonneuve-Rosemont and Université de Montréal, Montréal, Que.; the Geriatrics and Neurosciences Centre, Department of Medicine (Garcia), Queen's University, Kingston, Ont.; the Division of Neurology, Department of Medicine, University of Toronto, and the Rotman Research Institute Baycrest Centre for Geriatric Care (Chow), Toronto, Ont.; the Division of Geriatric Medicine, Department of Medicine, University of Western Ontario, and the Aging Brain and Memory Clinic, St. Joseph's Health Care - Parkwood Site (Borrie), London, Ont.; the Departments of Neurology and Neurosurgery and of Medicine (Schipper, Chertkow), McGill University and Sir Mortimer B. Davis-Jewish General Hospital, Montréal, Que.; and the Department of Clinical Neurological Sciences (Blair, Kertesz), University of Western Ontario, London, Ont.

This series is based on recommendations from the Third Canadian Consensus Conference on the Diagnosis and Treatment of Dementia.

Series editor: Howard Chertkow MD, Department of Neurology, McGill University and the Bloomfield Centre for Research in Aging, Lady Davis Institute, Sir Mortimer B. Davis-Jewish General Hospital, Montréal, Que. 
that his mother-in-law developed dementia at age 8o. Findings on physical examination are negative aside from Mrs. E's decreased cognitive abilities, which include disorientation to date and day of the week. She scores 24 out of 30 on the Mini-Mental State Examination, losing points for temporal orientation, short-term recall of test items and naming of test objects. Mrs. E's daughter asks you if her mother has Alzheimer disease.

What further diagnostic work-up should you perform to establish the diagnosis?

$\mathrm{T}$ his hypothetical case highlights a range of issues that commonly emerge in the diagnosis of a mild dementia. Cognitive impairment and dementia are present in about $20 \%$ of the elderly population and are consistently rated among the top 3 concerns of elderly people. ${ }^{1}$ Accurately diagnosing dementia remains a challenge for family physicians and specialists. There are 5 reasons why family physicians in Canada should develop expertise in dementia diagnosis. First, increasing numbers of elderly patients will be presenting with memory complaints to their family physicians. ${ }^{1}$ Second, current planning for geriatric and neurologic services in Canada works under the assumption that the burden of dementia diagnosis will largely fall on the shoulders of family physicians. Third, there continue to be a small subset of treatable disorders presenting as memory loss that must not be missed. Fourth, planning by families of elderly patients is most effective when dementia is diagnosed early in the illness. Fifth, symptomatic therapy that exists for Alzheimer disease is underused, alternative approaches are necessary for other conditions such as vascular dementia and frontotemporal dementia, and the list of therapies specific for different dementia diagnoses is likely to grow in the future. Accurate diagnosis is the prerequisite for optimal therapy.

There has been a wealth of research into the role and utility of different diagnostic methods over the past decade. The work has led to the development of better cognitive screening tools and more specific (and often expensive) means of diagnosing Alzheimer disease. Although numerous novel ap-

Box 1: Recommendations for the diagnosis of dementia* (part 1 of 2)

Brief cognitive tests

- A range of brief cognitive tests, including the Montréal Cognitive Assessment, ${ }^{2}$ the DemTect, ${ }^{3}$ the 7 -Minute Screen, ${ }^{4}$ the General Practitioner Assessment of Cognition ${ }^{5}$ and the Behavioural Neurology Assessment Short Form, ${ }^{6}$ may be more accurate than the Mini-Mental State Examination in discriminating between dementia and the normal state. There is insufficient evidence to recommend one test over the others [grade B recommendation, level 2 evidence; new recommendation].

- Brief cognitive tests have not been developed to differentiate between dementia subtypes and should not be used for this purpose [grade $D$ recommendation, level 2 evidence; new recommendation].

Clinical diagnosis

- The diagnosis of dementia remains clinical. There is good evidence to retain the diagnostic criteria currently in use ${ }^{7}$ [grade $\mathrm{A}$ recommendation, level 2 evidence; new recommendation].

- The sensitivity of clinical diagnosis for possible or probable Alzheimer disease based on the NINCDS-ADRDA criteriat remains high. The specificity is lower. The continued use of the NINCDS-ADRDA criteria is recommended [grade A recommendation, level 1 evidence; new recommendation].

- "Mild" Alzheimer disease can be diagnosed with a high degree of specificity, when the presenting clinical picture is one of memory impairment [grade $B$ recommendation, level 1 evidence; new recommendation].

\section{Laboratory investigations}

- For all patients who have a clinical presentation consistent with Alzheimer disease with typical cognitive symptoms or presentation, only a basic set of laboratory tests should be ordered to rule out causes of chronic metabolic encephalopathy producing chronic confusion and memory loss [grade B recommendation, level 3 evidence; recommendation unchanged].

- Complete blood count (to rule out anemia)

Thyroid stimulating hormone (to rule out hypothyroidism)

Serum electrolytes (to rule out hyponatremia)

Serum calcium (to rule out hypercalcemia)

Serum fasting glucose (to rule out hyperglycemia)

- The serum vitamin $B_{12}$ level should be determined in all older adults suspected of having dementia or cognitive decline [grade $B$ recommendation, level 2 evidence; new recommendation].

- Older adults found to have a low vitamin $B_{12}$ level should be given vitamin $B_{12}$ (either orally or parenterally) because of potential improvement of cognitive function and the deleterious effects of low vitamin $B_{12}$ levels on multiple organ systems, besides the effects on cognition [grade $B$ recommendation, level 2 evidence; new recommendation].

- Determination of serum folic acid or red blood cell folate levels in older adults in Canada is optional and may be reserved for patients with celiac disease, inadequate diet or other condition that prevents them from ingesting grain products [grade $\mathrm{E}$ recommendation, level 2 evidence; new recommendation].

- There is currently insufficient evidence to support the need for the determination of serum homocysteine levels in older adults with suspected dementia or cognitive decline [grade C recommendation, level 3 evidence; new recommendation].

- There is currently insufficient evidence that treatment of elevated serum homocysteine levels affects cognition [grade $C$ recommendation, level 3 evidence; new recommendation]. 
proaches have reached the mainstream media and received wide publicity, much confusion exists over which methods are sufficiently valid, reliable, available and inexpensive enough to warrant wide use by family physicians.

In this article, we provide physicians with practical guidance on the diagnosis of dementia based on evidence-based recommendations from the Third Canadian Consensus Conference on the Diagnosis and Treatment of Dementia (Box I). A description of the process used to generate the recommendations is provided in the first article of this series ${ }^{1}$ and in an online appendix accompanying this article (available at www .cmaj.ca/cgi/content/full/178/7/825/DC2). Published background papers provide details of the evidence-based reviews on which the recommendations are based..$^{8-13}$

\section{Approach to diagnosis}

The diagnostic process in dementia has 3 major conceptual components: the clinical diagnosis, a logical search for the cause, and the identification of treatable comorbid conditions and other contributing factors, such as the degree of cerebrovascular disease. The diagnostic process should involve 6 main steps: taking the patient's history, interviewing a caregiver or family member, physical examination, brief cognitive tests, basic laboratory tests, and structural imaging for patients meeting certain criteria. The conclusion involves meeting with the patient and his or her family to discuss the results and diagnosis, and their implications. Practically speaking, the diagnosis is rarely "urgent," and these steps are best carried out during repeated visits with the family practitioner over the course of weeks. Families should be alerted that this is an important process that warrants their time and effort. Most provinces have billing codes to allow adequate reimbursement of such assessments when spread over an extended period. In the following sections, we will outline these steps in more detail and address a number of other ancillary diagnostic methods, specifically functional imaging, neuropsychological testing, and measurement of blood and cere-

Box 1: Recommendations for the diagnosis of dementia* (part 2 of 2)

- Genetic testing, including screening for the apolipoprotein E gene, is not recommended for the purpose of diagnosing Alzheimer disease because the positive and negative predictive values are low [grade $\mathrm{E}$ recommendation, level 2 evidence; new recommendation].

Neuroimaging with computed tomography and magnetic resonance imaging

- Cranial computed tomography scanning is recommended if one or more of the following criteria are present [grade B recommendation, level 3 evidence; recommendation unchanged]:

- Age $<60$ years

- Rapid (e.g., over 1-2 months) unexplained decline in cognition or function

Short duration of dementia (< 2 years)

Recent and significant head trauma

Unexplained neurologic symptoms (e.g., new onset of severe headache or seizures)

History of cancer (especially types that metastasize to the brain)

Use of anticoagulants or history of bleeding disorder

History of urinary incontinence and gait disorder early in the course of dementia (as may be found in normal pressure hydrocephalus)

Any new localizing sign (e.g., hemiparesis or a Babinski reflex)

- Unusual or atypical cognitive symptoms or presentation (e.g., progressive aphasia)

Gait disturbance

- There is fair evidence to support the use of structural neuroimaging with computed tomography or magnetic resonance imaging to rule in concomitant cerebrovascular disease that can affect patient management [grade B recommendation, level 2 evidence; new recommendation].

\section{Neuropsychological testing}

- The diagnosis and differential diagnosis of dementia is currently a clinically integrative one. Neuropsychological testing alone cannot be used for this purpose and should be used selectively in clinical settings [grade B recommendation, level 2 evidence; new recommendation].

- Neuropsychological testing may aid in:

addressing the distinction between normal aging, mild cognitive impairment or cognitive impairment without dementia, and early dementia [grade B recommendation, level 2 evidence; new recommendation];

addressing the risk of progression from mild cognitive impairment or cognitive impairment without dementia to dementia or Alzheimer disease [grade B recommendation, level 2 evidence; new recommendation]; and

determining the differential diagnosis of dementia and other syndromes of cognitive impairment [grade B recommendation, level 2 evidence; new recommendation].

*Based on recommendations from the Third Canadian Consensus Conference on Diagnosis and Treatment of Dementia, held in March 2006.

†The criteria of the National Institute of Neurological and Communicative Disorders and Stroke (NINCDS) and the Alzheimer's Disease and Related Disorders Association (ADRDA) are provided in Box 4. 
brospinal fluid biomarkers. These last 3 diagnostic methods are usually restricted to specialist practice, but it is not uncommon for families to request information from their family physicians about the latest test for Alzheimer disease.

\section{Clinical evaluation}

Despite the remarkable progress that has been made in the basic neurosciences elaborating the molecular genetics, pathophysiology and neurochemistry of the degenerative dementias, the diagnosis of dementia is one that is still made clinically in the office, with a history from the patient, collateral history from an informant, and a physical examination. This must be supplemented by a cognitive assessment. The sensitivity for each of these components in detecting dementia varies strongly with the degree of dementia, moderate dementia being far less challenging to detect than very mild dementia.

\section{Patient history}

The history taking should focus on the cadence of the illness (gradual and insidious in Alzheimer disease, stepwise in vascular dementia) and the relation to any vascular events such as stroke. Causes of dementia such as alcohol abuse and renal failure should be assessed. Vascular risk factors, including hypertension, diabetes mellitus, smoking history, family history of stroke and lipid status, should be ascertained. Risk factors such as family history of dementia or repetitive head trauma, and protective factors such as high education level, should also be ascertained.

\section{Family interview}

A separate history taken from a caregiver or family member with the patient absent is obligatory. It is necessary to ascertain whether the memory complaint represents a consistent change from the previous level of function. Frontotemporal dementia is characterized by early personality changes that the patient will almost invariably fail to note or report. Reports of "embarrassing behaviour" or even functional impairment will often not be mentioned by family members if the patient is present. Functional impairment should be directly assessed and significant impairment documented. This involves questioning the caregiver or family member about the patient's independent performance of activities of daily living, such as feeding and toileting. In early dementia, the functional impairment is more likely to emerge in "higher" functions, such as the ability to carry out complicated financial affairs such as banking, the ability to use public transport or to drive, normal attention to hobbies, and the ability to learn to use new machines or appliances. ${ }^{14}$ This interview is also an opportunity to gauge family support systems and the social setting, which may prove crucial in the future management of the patient. A separate visit may often be arranged for such a family interview.

\section{Physical examination}

During the physical examination, the physician should pay particular attention to the potential signs of stroke, including hyperreflexia, extensor plantar responses, frontal gait apraxia and pseudobulbar palsy. The presence of small-vessel is- chemic cerebrovascular disease with concurrent senile neuritic plaques and neurofibrillary tangles increases the risk of dementia by an estimated 20 times. ${ }^{15}$

\section{Brief cognitive tests}

All patients evaluated for dementia should have their cognitive function evaluated. Brief cognitive tests serve to determine the presence and overall severity of memory and cognitive deficits and can be recommended for both primary care and specialty practice (Table I). They have grown to be part of the lexicon of communication across health disciplines caring for individuals with dementia. All such tests are a trade-off between the practical need for brevity and the importance of reliably documenting impairment across a set of cognitive domains, as is required for a clinical diagnosis of dementia. In essence, all brief cognitive tests have a lower sensitivity and specificity than does a full neuropsychological evaluation, but they are far faster and more accessible than specialized testing.

The Mini-Mental State Examination (MMSE) ${ }^{19}$ remains the most widely used instrument, with high sensitivity and specificity for separating moderate dementia from normal cognition. It requires little training, is administered in about to minutes and has vast medical acceptance. A rough rule of thumb is that patients with mild dementia usually have a score of $18-26$ out of 30 , those with moderate dementia a score of IO-I8, and those with severe dementia a score of less than Io. Some clinical trials of Alzheimer disease have accepted only patients with a score of less than $24 \cdot{ }^{16}$ At scores above this level, the Mini-Mental State Examination lacks sensitivity for the diagnosis of mild dementia, and other assessments are needed. The Mini-Mental State Examination focuses on memory, attention, construction and orientation domains. The Modified Mini-Mental State Examination is a more expanded version that also includes assessment of delayed recall ${ }^{20}$ and has been widely used in clinical and research settings in Canada.

The clock-drawing test evaluates general executive functioning of the frontal lobe, as well as visuospatial abilities (Figure I) ${ }^{22}$ It requires ${ }^{5}$-Io minutes to administer and has achieved widespread clinical use. Like the Mini-Mental State Examination, however, the clock-drawing test may lack sensitivity for the diagnosis of early or mild dementia. ${ }^{18}$

A number of newer tests have been developed to provide improved sensitivity. Of these, it is worth mentioning the Montréal Cognitive Assessment, ${ }^{2}$ DemTect, ${ }^{3}$ the 7 -Minute Screen, ${ }^{4}$ the General Practitioner Assessment of Cognition ${ }^{5}$ and the Behavioural Neurology Assessment short form. ${ }^{6}$ All of these tests have been shown to be more accurate than the Mini-Mental State Examination in discriminating between dementia and normal cognition, particularly in cases of very mild dementia. ${ }^{2-6}$ The General Practitioner Assessment of Cognition ${ }^{5}$ requires $5^{-}$-Io minutes to administer and was evaluated in family practice offices in Australia. The other tests require about Io or more minutes to administer, were evaluated in specialty clinics or population studies and are used to evaluate multiple cognitive domains. This coverage probably makes them more accurate in detecting dementia in hetero- 
geneous populations, but this remains to be proven. Although consideration of the details of these tests is beyond the scope of our review, interested readers may refer to the background paper by Jacova and colleagues ${ }^{3}$ on neuropsychological testing and brief cognitive testing (see the Editor's Note at the end of the article for information on how to obtain a copy). We lack clear knowledge of the advantages of one brief cognitive test over the others. At this point, the best advice is for physicians to gain experience and familiarity with one test and to use it routinely for diagnosing dementia.

\section{Does the patient meet criteria for dementia?}

At this point in the evaluation, the physician has accumulated sufficient information to decide whether the patient meets clinical criteria for dementia (Box 2). There is good evidence that clinical diagnostic criteria for dementia can be readily applied at the bedside. Well-validated criteria such as the Diagnostic and Statistical Manual of Mental Disorders, third edition, revised (DSM-III-R), ${ }^{23}$ and fourth edition, text revision (DSM-IV-TR), ${ }^{7}$ form the most widely used diagnos- tic framework in Canada. Objective testing of memory and cognitive function is fundamental to the application of these diagnostic criteria.

The challenges in diagnosing dementia should not be understated. It can be difficult in some individuals with mild dementia to reliably demonstrate objective cognitive impairment as well as functional impairment. Some individuals without dementia can score low in the Mini-Mental State Examination, and only a score below 20 provides specific evidence for dementia. ${ }^{24}$ Conversely, dementia is possible even with a Mini-Mental State Examination score greater than $26 . .^{25}$ Furthermore, the score may vary by several points from one evaluation to the next. Language barriers, advanced age and low education can also confound the results and provide false-positive scores. A briefer test, such as the clock-drawing test, has the same challenges. No one brief cognitive test has been found to be superior over the others. No brief cognitive test has been developed to differentiate between subtypes of dementia, and none can be recommended for this purpose. ${ }^{26}$ Often the best recommendation is to repeat the testing on

Table 1: Brief cognitive screening tests to assist in the diagnosis of dementia

\begin{tabular}{|c|c|c|c|c|c|c|}
\hline Test & $\begin{array}{c}\text { Cognitive domains } \\
\text { evaluated }\end{array}$ & $\begin{array}{l}\text { Time to } \\
\text { administer, } \\
\text { min }\end{array}$ & $\begin{array}{l}\text { Score } \\
\text { range }\end{array}$ & Validation samples & $\begin{array}{l}\text { Accuracy } \\
\text { estimates }\end{array}$ & Comments \\
\hline $\begin{array}{l}\text { Mini-Mental } \\
\text { State } \\
\text { Examination } \\
\text { (MMSE) }\end{array}$ & $\begin{array}{l}\text { Memory (immediate recall), } \\
\text { orientation, attention, } \\
\text { language, visuo-construction, } \\
\text { praxis }\end{array}$ & $10-15$ & $0-30$ & $\begin{array}{l}\text { Community and clinic } \\
\text { samples: dementia } \\
\text { patients v. normal } \\
\text { controls }\end{array}$ & $\begin{array}{l}\text { Sensitivity } 44 \%-100 \%{ }^{16} \\
\text { Specificity } 46 \%-100 \%{ }^{16}\end{array}$ & $\begin{array}{l}\text { Widely used in research and } \\
\text { clinic settings but lacks } \\
\text { sensitivity to detect mild } \\
\text { cognitive impairment levels }\end{array}$ \\
\hline $\begin{array}{l}\text { Modified Mini- } \\
\text { Mental State } \\
\text { Examination }\end{array}$ & $\begin{array}{l}\text { Memory (immediate and } \\
\text { delayed recall), orientation, } \\
\text { attention, language, } \\
\text { visuo-construction, praxis }\end{array}$ & $20-25$ & $0-100$ & $\begin{array}{l}\text { Large community } \\
\text { sample: Alzheimer } \\
\text { patients v. normal } \\
\text { controls }\end{array}$ & $\begin{array}{l}\text { Area under the } \\
\text { curve } 0.93^{17}\end{array}$ & $\begin{array}{l}\text { Widely used in research and } \\
\text { clinic settings but lacks } \\
\text { sensitivity to detect mild } \\
\text { cognitive impairment levels }\end{array}$ \\
\hline $\begin{array}{l}\text { Clock-drawing } \\
\text { test }\end{array}$ & $\begin{array}{l}\text { Executive functioning, } \\
\text { visuo-construction }\end{array}$ & $5-10$ & $\begin{array}{l}0-4 \text { to } \\
0-20^{*}\end{array}$ & $\begin{array}{l}\text { Research clinic sample: } \\
\text { dementia patients } \\
\text { v. patients without } \\
\text { dementia }\end{array}$ & $\begin{array}{l}\text { Sensitivity } 20 \%-60 \%{ }^{18} \\
\text { Specificity } 60 \%-93 \%^{18}\end{array}$ & $\begin{array}{l}\text { Widely used in research and } \\
\text { clinic settings but lacks } \\
\text { sensitivity to detect mild } \\
\text { cognitive impairment levels }\end{array}$ \\
\hline $\begin{array}{l}\text { Montréal } \\
\text { Cognitive } \\
\text { Assessment }\end{array}$ & $\begin{array}{l}\text { Memory (immediate and } \\
\text { delayed recall), orientation, } \\
\text { attention, executive } \\
\text { functioning, language, } \\
\text { visuo-construction }\end{array}$ & $15-25$ & $0-30$ & $\begin{array}{l}\text { Clinic samples: } \\
\text { Alzheimer patients } \\
\text { v. normal controls }\end{array}$ & $\begin{array}{l}\text { Sensitivity } 100 \%^{2} \\
\text { Specificity } 87 \%^{2}\end{array}$ & $\begin{array}{l}\text { More sensitive than MMSE in } \\
\text { detecting Alzheimer disease } \\
\text { and mild cognitive } \\
\text { impairment }\end{array}$ \\
\hline DemTect & $\begin{array}{l}\text { Memory (immediate and } \\
\text { delayed recall), attention, } \\
\text { executive functioning, } \\
\text { language }\end{array}$ & $10-20$ & $0-18$ & $\begin{array}{l}\text { Clinic samples: } \\
\text { 1. Alzheimer patients } \\
\text { v. normal controls } \\
\text { 2. Alzheimer patients } \\
\text { v. patients with mild } \\
\text { cognitive impairment }\end{array}$ & $\begin{array}{l}\text { 1. Sensitivity } 100 \% \text {; } \\
\text { specificity } 92 \%^{3} \\
\text { 2. Sensitivity } 85 \% \text {; } \\
\text { specificity } 81 \%^{3}\end{array}$ & $\begin{array}{l}\text { More sensitive than MMSE in } \\
\text { detecting Alzheimer disease } \\
\text { and mild cognitive impairment; } \\
\text { discriminates between } \\
\text { Alzheimer disease and mild } \\
\text { cognitive impairment }\end{array}$ \\
\hline $\begin{array}{l}\text { 7-Minute } \\
\text { Screen }\end{array}$ & $\begin{array}{l}\text { Memory (immediate and } \\
\text { delayed recall), } \\
\text { orientation, language, } \\
\text { visuo-construction }\end{array}$ & $10-15$ & $-†$ & $\begin{array}{l}\text { Clinic and community } \\
\text { samples: Alzheimer } \\
\text { patients v. normal } \\
\text { controls }\end{array}$ & $\begin{array}{l}\text { Sensitivity } 92 \%^{4} \\
\text { Specificity } 96 \%^{4}\end{array}$ & $\begin{array}{l}\text { More sensitive than MMSE in } \\
\text { detecting mild Alzheimer } \\
\text { disease; validated in primary } \\
\text { care setting }\end{array}$ \\
\hline $\begin{array}{l}\text { General } \\
\text { Practitioner } \\
\text { Assessment of } \\
\text { Cognition }\end{array}$ & $\begin{array}{l}\text { Memory (immediate and } \\
\text { delayed recall), } \\
\text { orientation, } \\
\text { visuo-construction, } \\
\text { activities of daily living }\end{array}$ & $5-10$ & $0-15$ & $\begin{array}{l}\text { Family physician office } \\
\text { sample: dementia } \\
\text { patients } v \text {. patients } \\
\text { without dementia }\end{array}$ & $\begin{array}{l}\text { Sensitivity } 82 \%-85 \%^{5} \\
\text { Specificity } 83 \%-86 \%^{5}\end{array}$ & $\begin{array}{l}\text { More sensitive and specific } \\
\text { than MMSE; developed and } \\
\text { validated for use by family } \\
\text { physicians; requires informant }\end{array}$ \\
\hline $\begin{array}{l}\text { Behavioural } \\
\text { Neurology } \\
\text { Assessment } \\
\text { Short Form }\end{array}$ & $\begin{array}{l}\text { Memory (immediate and } \\
\text { delayed recall), orientation, } \\
\text { attention, executive } \\
\text { functioning, language, } \\
\text { visuo-construction }\end{array}$ & $20-30$ & $0-114$ & $\begin{array}{l}\text { Clinic sample: } \\
\text { dementia patients } \\
\text { v. normal controls }\end{array}$ & $\begin{array}{l}\text { Sensitivity } 93 \%{ }^{6} \\
\text { Specificity } 93 \%^{6}\end{array}$ & $\begin{array}{l}\text { More sensitive than MMSE in } \\
\text { detecting dementia }\end{array}$ \\
\hline
\end{tabular}

*Multiple scoring methods are available that generate different score ranges.

†Subtest scores are entered into an algorithm to yield likelihood of dementia. 

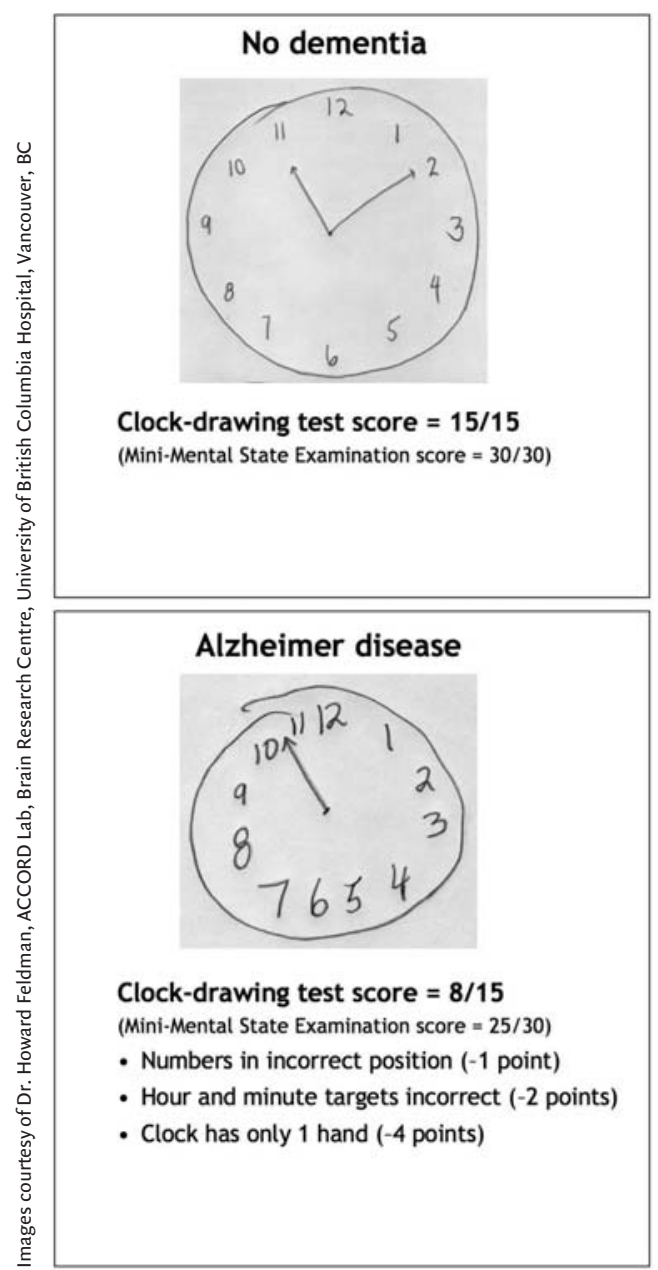

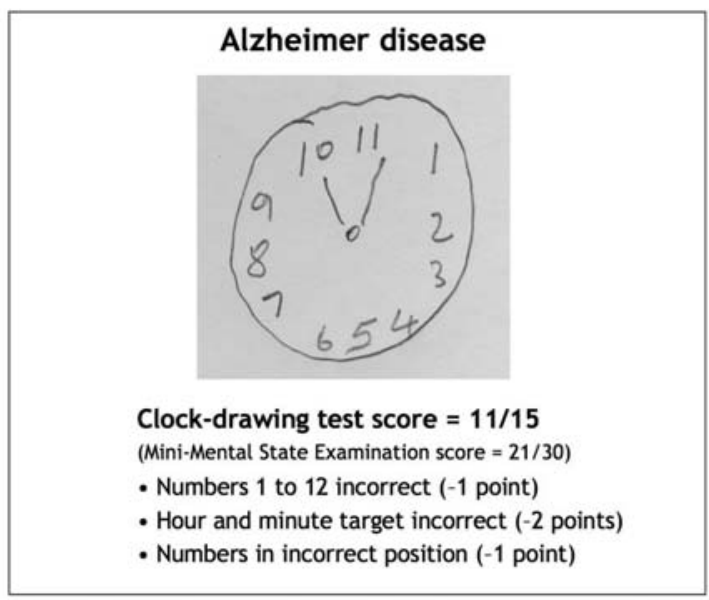

\section{Suspected frontotemporal dementia}

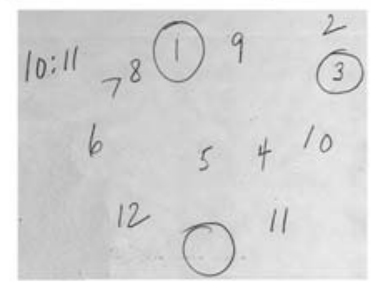

Clock-drawing test score $=3 / 15$

(Mini-Mental State Examination score $=25 / 30$ )

- No contour (-2 points)

- Superfluous markings (-1 point)

- Hour and minute targets incorrect (-2 points)

- Numbers in incorrect order / incorrect position / outside contour ( -3 points)

- Clock has no centre or hands / hands not joined / length of hour and minute hands cannot be compared ( -4 points)

\section{Scoring the clock drawing}

The clock drawing can be scored using the criteria described by Freedman and colleagues. ${ }^{21}$ Fifteen items are used to evaluate the drawing and 1 point is given for each item that is present. The following criteria are used - the shape of the circle is acceptable; it is not too small, overdrawn or repeated; only numbers 1 to 12 are present; all numbers are in Arabic numerals; the numbers are in the correct order; the paper is not rotated while drawing the numbers; the numbers are in the correct position; all numbers are inside the contour; the clock has a centre where the hands meet; the clock has 2 hands; the target number for the hour hand is indicated; the target number for minutes is indicated; the minute hand is longer than the hour hand; no superfluous markings are present; the hands are joined or are within $1.27 \mathrm{~cm}$ of each other.

Editor's Note: The original list that appears in the print version has been replaced with the above information.

Figure 1: Clock drawings and test scores for patients without dementia and those with Alzheimer disease or suspected frontotemporal dementia. Patients are instructed to draw a clock face with all the numbers in it, and to show the time as 10 minutes past 11 . The list of criteria used to determine a patient's score appears only in the print version of this article and is reproduced there from Freedman et al. ${ }^{21}$

several occasions over several months, to provide more convincing evidence of cognitive impairment. The documentation of functional impairment can also be challenging. If the patient had limited premorbid activities, it can be difficult to document convincing functional decline for several years.

\section{Differential diagnosis}

Having established the presence of a dementia, the specific cause should be determined. This determination relies on clinical evaluation, along with laboratory investigations and structural imaging (discussed in more detail in the next 2 sections). In considering diagnosis of a degenerative dementia, it is important to exclude delirium, a condition that is a transient, usually reversible, acute confusional state. Its clinical hallmarks are impaired attention and fluctuations in levels of consciousness. Delirium is a medical emergency with an increased risk for morbidity and death, and as such it is particularly significant in the differential diagnostic considerations. ${ }^{27-29}$ It calls for immediate investigation and management of treatable medical conditions. Individuals with dementia are at risk for delirium; however, the initial diagno- sis of dementia should not be made while the patient is in a delirious state. The most common risk factors and precipitating factors for delirium are listed in Box $3 .^{30}$

Depression and its contributing role in creating a "pseudodementia" has received considerable emphasis in the past. ${ }^{31}$ It is now appreciated that some degree of depressive symptoms, anxiety and apathy are quite common in the prodrome and course of Alzheimer disease. ${ }^{32,33}$ Asking the patient, or a family member or caregiver, about vegetative symptoms, including disorders of sleep, appetite and weight, can further address this diagnostic possibility of depression and the need for its treatment. Important symptoms to review include the presence of feelings of guilt, loss of enjoyment of pleasurable activities, current outlook and suicidal ideation.

The spectrum of dementias seen in patients referred to dementia clinics in Canada is provided in Table $2 .^{34}$ The significant majority of dementia referrals in this setting are clinically diagnosed as Alzheimer disease, either alone or in association with other comorbid conditions such as cerebrovascular disease, Parkinson disease or Lewy bodies. The aging brain is recognized to frequently play host to multiple pathologies. In 


\section{Box 2: Diagnostic criteria for dementia}

The presence of an acquired impairment in memory, associated with impairment in one or more cognitive domains, including:

- Executive function (e.g., abstract thinking, reasoning, judgment)

- Language (expressive or receptive)

- Praxis (learned motor sequences)

- Gnosis (ability to recognize objects, faces or other sensory information)

Impairments in cognition must be severe enough to interfere with work, usual social activities or relationships with others.

Source: Diagnostic and Statistical Manual of Mental Disorders, 4th edition, text revision.

particular, it has been increasingly appreciated that senile neuritic plaques and neurofibrillary tangles often exist with concurrent cerebrovascular lesions. ${ }^{35}$

In terms of clinical criteria for Alzheimer disease, the diagnostic criteria of the National Institute of Neurological and Communicative Disorders and Stroke and the Alzheimer's Disease and Related Disorders Association (NINCDS-ADRDA criteria) (Box 4) have been the most widely adapted for clinical use and have been neuropathologically validated. ${ }^{36,37}$

A brief description of the key characteristics of nonAlzheimer dementias is provided in Box 5 .

\section{Laboratory investigations}

The primary role of laboratory investigations and structural neuroimaging with computed tomography or magnetic resonance imaging is to rule out the rare presence of a treatable disorder presenting as memory loss, such as renal failure, brain tumour, normal pressure hydrocephalus and subdural hemorrhages. Recent studies have found such "treatable dementias" to account for less than $\mathrm{I} \%$ of dementia cases. ${ }^{38}$ The previous consensus conference on the assessment and treatment of dementia reviewed the best evidence to guide laboratory investigations. ${ }^{39}$ It recommended basic investigations for all patients, including complete blood count, thyroid stimulating hormone, and serum calcium, electrolytes and fasting glucose. Other laboratory tests were to be applied selectively based on an individual's presenting medical history, and cognitive and physical examination findings. Selective testing of serum vitamin $\mathrm{B}_{12}$ and folate levels, rapid plasma reagin for syphilis screening, and HIV antibodies were recommended.

The current recommendations for core laboratory investigations have not changed except for the measurement of serum $B_{12}$ levels, which is now recommended for all older adults who have suspected dementia or cognitive impairment (Box I). There is insufficient evidence to recommend testing of red blood cell folate or serum folic acid levels. Since the introduction of folic acid fortification of grain products in Canada in 1998, folate deficiencies are rare, and testing of red blood cell folate levels is recommended only if the patient has a history of inadequate diet, celiac disease or other conditions preventing the ingestion of grain products. The measurement of homocysteine levels has been a recent focus of attention, with epidemiologic studies suggesting that elevated levels may be a risk factor for Alzheimer disease and vascular dementia. There is insufficient evidence to recommend the measurement of homocysteine levels or the treatment of elevated levels. Genetic testing, including screening for the apolipoprotein $\mathrm{E}$ gene, is not recommended.

\section{Neuroimaging with computed tomography and magnetic resonance imaging}

The availability of neuroimaging with computed tomography or magnetic resonance imaging permits a detailed assessment of the structural patterns of brain atrophy, with particular attention to the medial temporal lobe, where the early atrophy in Alzheimer disease can be seen (Figure 2). In addition, neuroimaging allows exclusion of neurosurgical lesions, including tumours, subdural hematomas and hydrocephalus. The previous recommended indications for computed tomography scanning have not changed ${ }^{41}$ (Box I). The selective use of computed tomography as part of the work-up for dementia is still recommended.

There is fair evidence to add the indication of using computed tomography or magnetic resonance imaging to rule in concomitant cerebrovascular disease. It was not possible on the basis of the available evidence to separate the indications for computed tomography from those for magnetic resonance imaging.

Box 3: Common risk factors and precipitating factors for delirium

Risk factors

- Age $\geq 65$ yr

- Male sex

- Impaired cognition (dementia, cognitive impairment, history of delirium, depression)

- Impaired functional status (functional dependence, immobility, low level of activity, history of falls)

- Sensory impairment (visual or hearing)

- Poor nutritional status (dehydration, malnutrition)

- Drugs (polypharmacy, psychoactives, anticholinergics, alcohol abuse)

- Presence of medical comorbidity (severe illness, multiple coexisting conditions, chronic renal or hepatic disease, history of stroke, neurologic disease, metabolic disorders, fracture or trauma, terminal illness, HIV infection)

\section{Precipitating factors}

- Drugs (sedative hypnotics, narcotics, anticholinergics, polypharmacy, alcohol or drug withdrawal)

- Primary neurologic disease (stroke, intracranial hemorrhage, meningitis or encephalitis)

- Intercurrent illness

- Surgery (orthopedic, cardiac, other)

- Environment (admission to intensive care unit, use of physical restraints, bladder catheter, pain, emotional stress, prolonged sleep deprivation)

Adapted from Inouye. ${ }^{30}$ 
Table 2: Types of dementia seen in patients referred to dementia clinics in Canada

\begin{tabular}{lc}
\hline Type of dementia & \% of patients \\
\hline Alzheimer disease & 47.2 \\
Mixed Alzheimer disease & 27.5 \\
Mixed others & 6.3 \\
Vascular dementia & 8.7 \\
Frontotemporal dementia & 5.4 \\
Dementia associated with Parkinson & \\
disease or with Lewy bodies & 2.5 \\
Unclassifiable & 1.8 \\
Other & 0.7 \\
\hline
\end{tabular}

Source: Feldman et al. ${ }^{34}$

\section{Additional procedures for diagnosis}

\section{Functional neuroimaging}

In addition to imaging techniques that look at brain structure (computed tomography and magnetic resonance imaging), there are techniques that look at the function of brain tissue and can visualize brain activity in vivo. There has been significant recent progress in these techniques. Modalities of functional neuroimaging include positron emission tomography with fluoro-D-2-deoxyglucose, single photon emission computed tomography, functional magnetic resonance imaging and magnetic resonance spectroscopy. They vary from being widely available (single photon emission computed tomography) to being available only in research settings (magnetic resonance spectroscopy). None of these technologies is recommended for current routine diagnostic evaluation of dementia. Nevertheless, there is fair evidence that positron emission tomography or single photon emission computed tomography can assist specialists in diagnosing cases of questionable early dementia or in discriminating between frontotemporal dementia and Alzheimer disease.

\section{Neuropsychological testing}

Beyond the administration of brief cognitive tests, it is possible in many settings to refer patients for neuropsychological testing. However, such referrals should be made selectively in clinical settings ${ }^{42}$ (Box I). Neuropsychological testing routinely requires $2-4$ hours of the patient's time, costs between $\$ 600$ and $\$ 1500$ and is often not covered by provincial government insurance plans. Within hospitals, there can be long waiting times for a neuropsychological evaluation, which can also limit its utility in routine dementia diagnosis. The diagnosis and differential diagnosis of dementia remain a clinically integrative process, with neuropsychological testing providing supportive evidence only. Nevertheless, neuropsychological testing provides detailed, standardized assessment across a wide range of cognitive domains, and with expert interpretation it has shown utility in distinguishing early or mild dementia from mild cognitive impairment or cognitive impairment without dementia and from normal cognitive function. ${ }^{43-45}$ Neuropsychological testing has greater sensitiv- ity than brief cognitive tests in documenting clear cognitive abnormalities across a range of cognitive domains. (The topics of mild cognitive impairment and cognitive impairment without dementia will be covered extensively in the next article in the series.)

There is evidence that neuropsychological testing can contribute to determining the likelihood of future dementia in atrisk groups. ${ }^{46}$ Neuropsychological testing has demonstrated utility in distinguishing between dementia subtypes, for instance, in suggesting that there might be frontotemporal dementia rather than Alzheimer disease. ${ }^{47-49}$ In this sense, the impairment that is detected through neuropsychological testing has considerable specificity, far greater than brief cognitive testing.

\section{Measurement of biological markers}

A biological marker of a disease can be defined as a measurable change in the physical constitution of a host that indicates the presence of that disease. The successful development of a specific biomarker for Alzheimer disease that could distinguish Alzheimer disease from normal aging and other forms of dementia would represent a major advance. Although there has been definite progress along this path, there is no ideal biomarker currently available. There are rare genetic factors that are invariably causative of earlyonset familial Alzheimer disease. ${ }^{50}$ They do not play a role in the vast majority of cases of Alzheimer disease, which are sporadic. The leading biochemical biomarkers that have advanced most in research include cerebrospinal fluid levels of $\beta$-amyloid ${ }_{1-42},{ }^{51}$ total tau, ${ }^{52}$ and phospho-tau (ptau) proteins. ${ }^{53-55}$ Decreased levels of $\beta$-amyloid ${ }_{1-42}$ and increased levels of p-tau are currently the most accurate and reproducible chemical biomarkers for early-onset Alz-

Box 4: Diagnostic criteria for Alzheimer disease of the National Institute of Neurological and Communicative Disorders and Stroke and the Alzheimer's Disease and Related Disorders Association (NINCDS-ADRDA criteria)

- Dementia established by means of clinical diagnosis and cognitive testing

- Progressive worsening of memory and other cognitive functions

- No disturbance of consciousness

- Absence of systemic disorders or other brain diseases that could account for the progressive cognitive decline

- Supportive features include: Altered behavioural patterns

Family history of similar disorders, particularly if confirmed neuropathologically

- Features that make a diagnosis of Alzheimer disease uncertain or unlikely include:

Sudden onset

Focal neurologic findings, including hemiparesis, sensory loss, visual field deficits, incoordination

Early presence of a gait disorder or seizure

Adapted, with permission, from McKhann et al. ${ }^{36}$ 
heimer disease..$^{51,53-55}$ However, they require lumbar puncture, and laboratories for testing these biomarkers are not readily available in Canada. Currently, these cerebrospinal fluid biomarkers may have utility for specialists who seek to distinguish between frontal variants of Alzheimer disease and frontotemporal dementia. When indicated, testing for biomarkers should be performed at a commercial or academic facility with a track record of generating reproducible, high-quality data.

\section{Knowledge gaps}

In this article we have alluded to several relevant current gaps in our knowledge. We have stressed the centrality of "clinical assessment," but we must acknowledge that our clinical definitions have a historical basis rather than an empirical one. For instance, the importance of demonstrating "impaired day-to-day function" in dementia is largely a function of the tendency of psychiatric diagnoses to stress presence or lack of functional impairment. We lack proof that the definition of dementia noted above is superior to alternative ones, or that our definition of cognitive domains is optimal. We do not know exactly how accurate brief cognitive tests are in comparison with one another, or even which method of scoring tests, such as the clock-drawing test, is preferable. For instance, is it better to administer a shorter test (the clock-drawing test) at biannual visits rather than a longer test (the Mini-Mental State Examination) at an initial visit? The ultimate impact of applying different approaches to brief cognitive testing is unknown. It is also unknown exactly how many cases of reversible dementia would be missed in a family practice if structural neuroimaging was not used - the only data have been generated retrospectively in a tertiary care memory clinic. ${ }^{56} \mathrm{Re}-$ garding the ancillary tests (neuropsychological testing, functional imaging and measurement of biomarkers), we lack informed large-scale studies with a cost-benefit analysis sufficient to justify their general application. For functional imaging, there is variability across centres, which require requisite expertise for this technology to have diagnostic utility. ${ }^{57,58}$ Standardization of techniques is necessary for them to have diagnostic utility.

Perhaps more important than any of these knowledge gaps are the large gaps between what we know about diagnosis and what is practised in many family physicians' offices. Less than $25 \%$ of cases of Alzheimer disease in Canada are diagnosed and treated, ${ }^{59,60}$ which implies a lack of application of known diagnostic approaches. The major hurdles to diagnosing dementia in family practice are (a) the complexity of the diagnostic process, (b) physicians' lack of familiarity with cognitive screening, (c) the pressures of time (the diagnostic process requires multiple visits to complete) and (d) the lack of general conviction that an accurate diagnosis of dementia warrants the requisite effort. Physician education is needed to help address these hurdles, and we hope that this article will help to encourage family physicians to undertake the diagnostic process more routinely.

\section{The case revisited}

Mrs. E presents with a memory complaint corroborated by her family, along with clear historical evidence of a progressive cognitive decline with collateral information from her husband that her impairment has reached the point of interfering with her social functioning and activities of daily living. Her physician conducts the Mini-Mental State Examination as the objective brief cognitive test, and her score confirms the presence of memory impairment, as evidenced by her impaired learning of test objects and by her temporal disorientation. There is also mild impairment of language (naming). In her daily life, there is evidence of impaired executive functioning (inability to handle banking) and topographic disorientation (getting lost while driving). On this basis, Mrs. E meets the clinical definition of dementia.

The next step is to determine the cause of Mrs. E's dementia. Her physician works through a logical list of exclusions. There is no evidence of delirium. The depressive symptoms and anxiety require more exploration to determine their significance and their relationship to the cognitive impairment. However, from the available information, a confounding major depression or primary anxiety disorder is unlikely to be present. The physician reviews Mrs. E's medications, paying particular attention to the use of any psychoactive medications, including sedative hypnotics, antipsychotics and antidepressants as well as drugs with potential anticholinergic properties. None of these confounding disorders are identified.

Box 5: Key characteristics of non-Alzheimer disease dementias

\section{Frontotemporal dementia}

- Younger age of onset than Alzheimer disease

- Hallmark features typically include either:

Prominent behavioural changes (e.g., social conduct dysregulation, disinhibition, perseveration and emotional blunting) OR

Prominent language impairment (e.g., progressive nonfluent aphasia or semantic problems, with breakdown of word meaning and knowledge)

Dementia associated with Lewy bodies or with Parkinson disease

- Clinical features of these 2 conditions overlap considerably

- Dementia associated with Parkinson disease begins with idiopathic Parkinson disease for $\geq 1$ year before the onset of dementia, whereas dementia associated with Lewy bodies begins with a cognitive and behavioural disorder that can have concurrent parkinsonian features

- Neuropsychiatric features include visual hallucinations and fluctuations in disease course

\section{Vascular dementia}

- Typically evolves in stepwise fashion but can also progress insidiously

- Hallmark cognitive feature of a dysexecutive syndrome

- Focal neurologic findings frequently found early in the disease course 


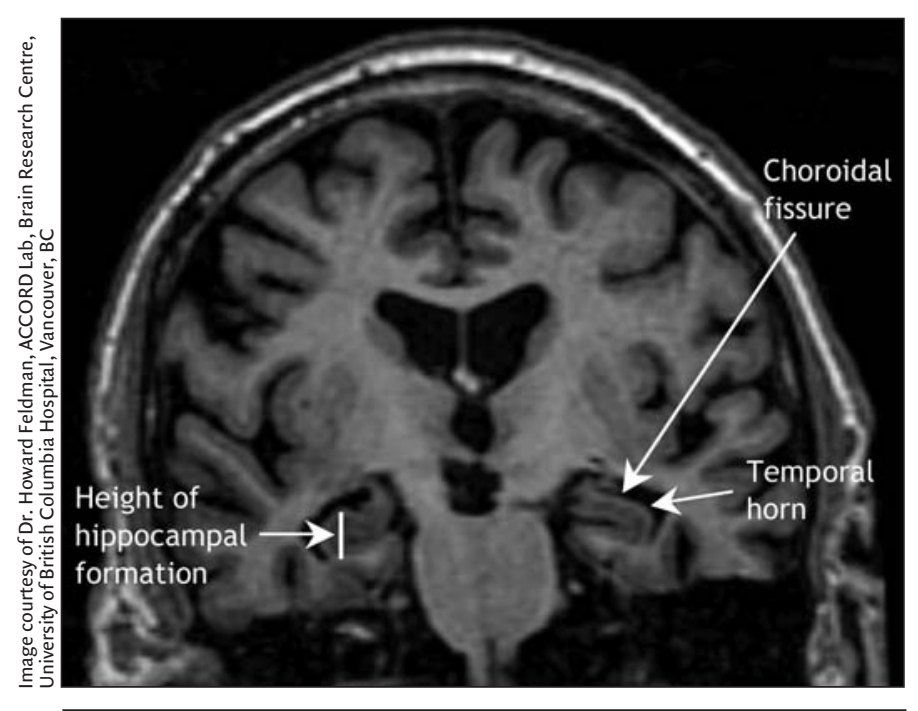

Figure 2: $T_{1}$-weighted coronal magnetic resonance imaging scan showing extensive hippocampal atrophy (arrows). These landmarks are based on a visual rating scale of medial temporal lobe atrophy developed by Scheltens and colleagues. ${ }^{40}$

In the continued search for the cause of her dementia, the physician notes the patient's history of a transient ischemic attack and considers a potential contributing cerebrovascular component to the dementia. A review of vascular risk factors and examination for signs of stroke would be appropriate. However, although the presence of a transient ischemic attack does bring up cerebrovascular considerations, the overall natural history is not one of stroke-like episodes with stepwise decline, or of a stroke followed within short order by a progressive dementia, as might be the case in a vascular dementia. ${ }^{61,62}$ On physical examination, Mrs. E's physician finds no focal neurologic signs. From a differential diagnosis standpoint, he finds no parkinsonian features, fluctuations in cognition and alertness, or visual hallucinations to suggest the core features of dementia associated with Parkinson's disease or dementia associated with Lewy bodies. ${ }^{63}$ Mrs. E has no myoclonus, and her course is not rapidly progressive as would typically be the case with Creutzfeld-Jakob disease. ${ }^{64}$ The lack of an early and prominent behavioural change with impairment in social conduct, progressive aphasia or motor neuron features puts aside consideration of frontotemporal dementia. The core and supportive features of Alzheimer disease are therefore met, and Mrs. E meets the clinical diagnostic criteria for Alzheimer disease.

Mrs. E's Mini-Mental State Examination score revealed impairment that was at the cut-off point for dementia. Her physician considers administering one of the newer brief cognitive tests, such as the Montréal Cognitive Assessment, the DemTect, the Behavioural Neurology Assessment, the General Practitioner Assessment of Cognition or the 7Minute Screen (Table I). These tests would likely demonstrate a greater degree of impairment, reinforcing the degree and significance of the cognitive impairment and its commensurate relationship with dementia. However, he decides not to because there is already considerable evidence of sufficient cognitive impairment (on temporal orientation, short-term recall and naming) and functional impairment (on history) to establish a diagnosis of dementia.

Mrs. E's physician orders a basic laboratory work-up, including a complete blood count and serum levels of calcium, thyroid stimulating hormone, fasting serum glucose and vita$\min B_{12}$. Beyond the investigations required for dementia diagnosis, her physician reviews her history of transient ischemic attack to ensure that measures for secondary stroke prevention are in place.

Because of Mrs. E's history of transient ischemic attack and lack of prior neuroimaging, her physician will request that she undergo computed tomography or magnetic resonance imaging to rule in cerebrovascular disease. This approach is particularly focused on the patient with cerebrovascular risk factors, clinical stroke or transient ischemic attack episodes. The scans will allow her physician to assess the extent, location and contribution of underlying cerebrovascular disease to her Alzheimer dementia and may guide the intensity of treatment of her vascular risk factors. There is no indication for functional neuroimaging or neuropsychological testing given the strength of the clinical diagnosis. There are no signs of frontotemporal dementia or atypical features to support the use of lumbar puncture for the measurement of $\beta$-amyloid - $_{1-42}$ protein, total tau protein and p-tau protein levels.

In summary, Mrs. E was judged on clinical grounds to meet the diagnostic criteria for dementia. Her presentation is most consistent with Alzheimer disease, given the gradual onset of a progressive cognitive decline without any highlighting strokes, stepwise decline or clinical features to suggest an alternative diagnosis. The use of neuropsychological testing would clarify the cause of the dementia; however, in this instance it is not required. The physician schedules a further visit with the family after all the results are back so that he can describe the diagnostic results, give the diagnosis of Alzheimer disease, discuss the prognosis and initiate therapy. These management issues will be addressed in a further article in this series.

\section{Conclusion}

The diagnosis of dementia remains clinically integrative based on history, physical examination and cognitive assessment. Brief cognitive testing is indicated for each patient presenting for assessment of dementia. Recommended investigations also include a number of core laboratory tests. Structural neuroimaging with computed tomography or magnetic resonance imaging is advised on a selective basis according to clinical features and the natural history of the presentation.

There are a growing set of promising diagnostic approaches that families of patients will have encountered in the media but that are not yet recommended for routine use by family physicians. Although functional neuroimaging with positron emission tomography or single photon emission computed tomography is evolving rapidly, application of these technologies to the clinical investigation of dementia is still limited. Similarly, although the measurement of bio- 
markers is not yet ready for widespread use in clinical settings, it is showing promise in the research setting and may change our approach to dementia evaluation in the near future. Neuropsychological testing is powerful but expensive and not readily available to family physicians in Canada. It is not essential in the routine evaluation of dementia patients.

\section{This article has been peer reviewed.}

Competing interests: None declared for Howard Feldman, Claudia Jacova, Angeles Garcia, Tiffany Chow, Michael Borrie, Mervin Blair or Howard Chertkow. Alain Robillard has been a member of the speaker's board, has served as a consultant to or has given lectures sponsored by Janssen-Ortho, Novartis, Pfizer and Lundbeck; he has received travel assistance to attend scientific meetings by Novartis, Pfizer and Janssen-Ortho; and he is the principal investigator for a number of clinical trials sponsored by the previously named companies as well as Elan Pharmaceuticals, Sanofi Aventis, Voyager Pharmaceutical, SmithKline Beecham and Ono Pharmaceutical. Tiffany Chow has served as a consultant to Janssen-Ortho and has received speaker fees or educational grants from Novartis and Lundbeck. Hyman Schipper has served as a consultant to Osta Biotechnologies, Teva Neurosciences and Caprion Pharmaceuticals; he holds equity in Molecular Biometrics and stock options in Osta Biotechnologies. Andrew Kertesz has served as a consultant and has received honoraria, speaker fees and travel assistance from Pfizer, Janssen-Ortho, Novartis and Lundbeck.

Contributors: All of the authors contributed to the study conception and design, revised the manuscript critically for important intellectual content and approved the final version submitted for publication.

Acknowledgements: We gratefully acknowledge the expert technical assistance of Jacob Grand in developing this paper and Dr. Najeeb Qadi for his help with the references.

Editor's Note: The background papers with supporting evidence for the recommendations from the Third Canadian Consensus Conference on the Diagnosis and Treatment of Dementia were published in the October 2007 issue of Alzheimer's and Dementia and are available at www.alzheimersanddementia.org. These articles are also freely available at www.cccdtd.ca (through agreement with Elsevier).

\section{REFERENCES}

I. Chertkow H. Diagnosis and treatment of dementia: Introduction. Introducing a series based on the Third Canadian Consensus Conference on the Diagnosis and Treatment of Dementia. CMAJ 2008;178:316-2I.

2. Nasreddine ZS, Phillips NA, Bedirian V. The Montreal Cognitive Assessment MoCA: a brief screening tool for mild cognitive impairment. J Am Geriatr Soc 2005;53:695-9

3. Kalbe E, Kessler J, Calabrese P, et al. DemTect: a new, sensitive cognitive screening test to support the diagnosis of mild cognitive impairment and early dementia. Int J Geriatr Psychiatry 2004;19:136-43.

4. Solomon PR, Hirschoft A, Kelly B, et al. A 7 minute neurocognitive screening battery highly sensitive to Alzheimer's disease. Arch Neurol 1998;55:349-55

5. Brodaty H, Pond D, Kemp NM, et al. The GPCOG: a new screening test for dementia designed for general practice. J Am Geriatr Soc 2002;50:530-4.

6. Darvesh S, Leach L, Black SE, et al. The behavioural neurology assessment. Can J Neurol Sci 2005;32:167-77.

7. American Psychiatric Association. Diagnostic and statistical manual of mental disorders. 4 th ed. Text revision. Washington (DC): The Association; 2000

8. Robillard A. Clinical diagnosis of dementia. Alzheimers Dement 2007;4:292-8.

9. Jacova C, Kertesz A, Blair M, et al. Neuropsychological testing and assessment for dementia. Alzheimers Dement 2007;4:299-317.

Io. Schipper HM. The role of biologic markers in the diagnosis of Alzheimer's disease. Alzheimers Dement 2007;4:325-32.

II. Chow T. Structural neuroimaging in the diagnosis of dementia. Alzheimers Dement 2007;4:333-5.

I2. Borrie M. Functional neuroimaging in the diagnosis of dementia. Alzheimers Dement 2007:4:336-40.

13. Garcia A. Cobalamin and homocysteine in older adults: Do we need to test for serum levels in the work-up of dementia? Alzheimers Dement 2007;4:318-24.

I4. Galvin JE, Roe CM, Powlishta KK, et al. The AD8: a brief informant interview to detect dementia. Neurology 2005;65:559-64
I5. Snowdon DA, Greiner LH, Mortimer JA, et al. Brain infarction and the clinical expression of Alzheimer disease. The Nun Study. JAMA 1997;277:813-7.

I6. Tombaugh TN, McIntyre NJ. The Mini-Mental State Examination: a comprehensive review. J Am Geriatr Soc I992;40:922-35.

17. Tombaugh TN, McDowell I, Kristjansson B, et al. Mini-Mental State Examination (MMSE) and the Modified MMSE (3MS): a psychometric comparison and normative data. Psychol Assess I996;8:48-59.

I8. Powlishta KK, Von Dras DD, Stanford A, et al. The clock drawing test is a poor screen for very mild dementia. Neurology 2002;59:898-903.

I9. Folstein MF, Folstein SE, McHugh PR. Mini-Mental State: a practical method for grading the state of patients for the clinician. JPsychiatr Res 1975;12:189-08.

20. Teng EL, Chui HC. The Modified Mini-Mental State (3MS) examination. J Clin Psychiatry $1987 ; 48: 314-8$.

21. Freedman M, Kaplan E, Delis D, et al. Clock drawing: a neuropsychological analysis. Oxford (UK): Oxford University Press; I994.

22. Shulman KI. Clock-drawing: Is it the ideal cognitive screening test? Int J Geriatr Psychiatry 2000;15:548-6I.

23. American Psychiatric Association. Diagnostic and statistical manual of mental disorders. 3 rd ed. Revised. Washington (DC): The Association; I987.

24. Siu AL. Screening for dementia and investigating its causes [review]. Ann Intern Med I99I;II5:I22-32.

25. Shiroky JS, Schipper HM, Bergman H, et al. Can you have dementia with a MMSE score of thirty? Am J Alzheimers Dis Other Demen 2007;22:406-I5.

26. Stuss DT, Meiran N, Guzman DA, et al. Do long tests yield a more accurate diagnosis of dementia than short tests? A comparison of 5 neuropsychological tests. Arch Neurol I996;53:1033-9.

27. Young J, Inouye SK. Delirium in older people. BMJ 2007;334:842-6.

28. Bhat R, Rockwood K. Delirium as a disorder of consciousness. J Neurol Neurosurg Psychiatry. 2007 May 8; [Epub ahead of print].

29. Liptzin B, Levkoff SE, Gottlieb GL. Delirium. J Neuropsychiatry Clin Neurosci 1993;5:154-60.

30. Inouye SK. Delirium in older persons [erratum in N Engl J Med 2006;354:I655]. N Engl J Med 2006;354:1157-65.

3I. Dobie DJ. Depression, dementia, and pseudodementia. Semin Clin Neuropsychiatry 2002;7:170-86.

32. Feldman H, Scheltens P, Scarpini E, et al. Behavioral symptoms in mild cognitive impairment. Neurology 2004;62:1199-20I.

33. Mega MS, Cummings JL, Fiorello T, et al. The spectrum of behavioural changes in Alzheimer's disease. Neurology I996;46:130-5.

34. Feldman H, Levy AR, Hsiung G-Y, et al. A Canadian Cohort Study of Cognitive Impairment and Related Dementias (ACCORD): study methods and baseline results. Neuroepidemiology 2003;22:265-74.

35. Jellinger KA, Attems J. Prevalence and pathological role of cerebrovascular lesions in Alzheimer disease. J Neurol Sci 2005;229-230:37-4I.

36. McKhann G, Drachman D, Folstein M, et al. Clinical diagnosis of Alzheimer's disease: report of the NINCDS-ADRDA Work Group under the auspices of Department of Health and Human Services Task Force on Alzheimer's Disease. Neurology I984;34:939-44.

37. Blacker D, Albert MS, Bassett SS, et al. Reliability and validity of NINCDS-ADRDA criteria for Alzheimer's disease. The National Institute of Mental Health Genetics Initiative. Arch Neurol I994;51:II98-204.

38. Clarfield AM. The reversible dementias: Do they reverse? Ann Intern Med I988; Io9: 476-86.

39. Patterson CJ, Gauthier S, Bergman H, et al. Canadian Consensus Conference on Dementia: a physician's guide to using the recommendations. CMAJ I999; I60: I738-42.

40. Scheltens P, Leys D, Barkhof F, et al. Atrophy of medial temporal lobes on MRI in "probable" Alzheimer's disease and normal ageing: diagnostic value and neuropsychological correlates. JNeurol Neurosurg Psychiatry i 992;55:967-72.

4I. Patterson C, Gauthier S, Bergman H, et al. The recognition, assessment and management of dementing disorders: conclusions from the Canadian Consensus Conference on Dementia. Can J Neurol Sci 200I;28(Suppl I):S3-I6.

42. Chertkow H, Bergman H, Schipper HM, et al. Assessment of suspected dementia. Can J Neurol Sci 200I;28(Suppl I):S28-4I.

43. Petersen RC, Smith GE, Waring SC, et al. Mild cognitive impairment: clinical characterization and outcome. Arch Neurol 1999;56:303-8.

44. Steenhuis RE, Ostbye T. Neuropsychological test performance of specific diagnostic groups in the Canadian Study of Health and Aging (CSHA). J Clin Exp Neuropsychol I995; 17:773-85.

45. Lambon Ralph MA, Patterson K, Graham N, et al. Homogeneity and heterogeneity in mild cognitive impairment and Alzheimer's disease: a cross-sectional and longitudinal study of 55 cases. Brain 2003;126:2350-62.

46. Backman L, Jones S, Berger AK, et al. Cognitive impairment in preclinical Alzheimer's disease: a meta-analysis. Neuropsychology 2005;19:520-31.

47. Ballard CG, Ayre G, O'Brien J, et al. Simple standardised neuropsychological assessments aid in the differential diagnosis of dementia with Lewy bodies from Alzheimer's disease and vascular dementia. Dement Geriatr Cogn Disord i999; I0: I04-8.

48. Tierney MC, Black SE, Szalai JP, et al. Recognition memory and verbal fluency differentiate probable Alzheimer disease from subcortical ischemic vascular dementia. Arch Neurol 2001; 58:1654-9.

49. Kertesz A, Davidson W, McCabe P, et al. Behavioral quantitation is more sensitive than cognitive testing in frontotemporal dementia. Alzheimer Dis Assoc Disord 2003;17:223-9. 
50. Patterson C, Feightner JW, Garcia A, et al. Diagnosis and treatment of dementia: I. Risk assessment and primary prevention of Alzheimer disease. CMAJ 2008;178: 548-56.

5I. Iwatsubo T. Amyloid beta protein in plasma as a diagnostic marker for Alzheimer's disease. Neurobiol Aging I998; I9:I6I-3

52. Sunderland T, Linker G, Mirza N, et al. Decreased beta-amyloidi- 42 and increased tau levels in cerebrospinal fluid of patients with Alzheimer disease. JAMA 2003;289:2094-103.

53. Blennow K, Hampel H. CSF markers for incipient Alzheimer's disease. Lancet Neurol 2003;2:605-13.

54. Hampel H, Buerger K, Zinkowski R, et al. Measurement of phosphorylated tau epitopes in the differential diagnosis of Alzheimer disease: a comparative cerebrospinal fluid study. Arch Gen Psychiatry 2004;61:95-102.

55. Lewczuk P, Esselmann H, Bibl M, et al. Tau protein phosphorylated at threonine I8I in CSF as a neurochemical biomarker in Alzheimer's disease: original data and review of the literature. J Mol Neurosci 2004;23:115-22.

56. Freter S, Bergman H, Gold S, et al. Prevalence of potentially reversible dementias and actual reversibility in a memory clinic cohort. CMAJ I998;159:657-62.

57. Mosconi L. Brain glucose metabolism in the early and specific diagnosis of Alzheimer's disease. FDG-PET studies in MCI and Alzheimer's disease. Eur J Nucl Med Mol Imaging 2005;32:486-510.

58. Dougall NJ, Bruggink S, Ebmeier KP. Systematic review of the diagnostic accuracy of 99mTc-HMPAO-SPECT in dementia. Am J Geriatr Psychiatry 2004;12:554-70.

59. Sternberg SA, Wolfson C, Baumgarten M. Undetected dementia in communitydwelling older people: the Canadian Study of Health and Aging. J Am Geriatrics Soc 2000;48:1430-4.

6o. Callahan CM, Hendrie HC, Tierney WM. Documentation and evaluation of cognitive impairment in elderly primary care patients. Ann Intern Med I995;I22:422-9.

6r. Roman GC, Tatemichi TK, Erkinjuntti T, et al. Vascular dementia: diagnostic criteria for research studies. Report of the NINDS-AIREN International Workshop. Neurology 1993;43:250-60.
62. Hachinski VC, Iliff LD, Zilhka E, et al. Cerebral blood flow in dementia. Arch Neurol I975;32:632-7.

63. McKeith IG, Dickson DN, Lowe J, et al. Diagnosis and management of dementia with Lewy bodies: third report of the DLB Consortium. Neurology 2005;65:1863-72.

64. Brown P, Gibbs CJ, Rodgers-Johnson P, et al. Human spongiform encephalopathy: the National Institutes of Health series of 300 cases of experimentally transmitted disease. Ann Neurol 1994;35:513-29.

Correspondence to: Dr. Howard H. Feldman, Division of Neurology, University of British Columbia Hospital, Sig2-22II Wesbrook Mall, Vancouver BC V6T 2B5; fax 604 822-7703; hfeldman@interchange.ubc.ca

\section{Articles to date in this series}

- Chertkow H. Diagnosis and treatment of dementia: Introduction. Introducing a series based on the Third Canadian Consensus Conference on the Diagnosis and Treatment of Dementia. CMAJ 2008;178:316-21.

- Patterson C, Feightner JW, Garcia A, et al. Diagnosis and treatment of dementia: 1 . Risk assessment and primary prevention of Alzheimer disease. CMAJ 2008;178:548-56. 